PROCEEDINGS OF THE

AMERICAN MATHEMATICAL SOCIETY

Volume 126, Number 6, June 1998, Pages 1765-1768

S $0002-9939(98) 04286-5$

\title{
ON ASYMMETRY OF TOPOLOGICAL CENTERS OF THE SECOND DUALS OF BANACH ALGEBRAS
}

\author{
F. GHAHRAMANI, J. P. MCCLURE, AND M. MENG
}

(Communicated by Theodore W. Gamelin)

\begin{abstract}
Let $\mathfrak{A}$ be a Banach algebra with a bounded approximate identity and let $Z_{1}\left(\mathfrak{A}^{* *}\right)$ and $Z_{2}\left(\mathfrak{A}^{* *}\right)$ be the left and right topological centers of $\mathfrak{A}^{* *}$. It is shown that i) $\mathfrak{A}^{*} \mathfrak{A}=\mathfrak{A A}^{*}$ is not sufficient for $Z_{1}\left(\mathfrak{A}^{* *}\right)=Z_{2}\left(\mathfrak{A}^{* *}\right)$; ii) the inclusion $\hat{\mathfrak{A}} Z_{1}\left(\mathfrak{A}^{* *}\right) \subseteq \hat{\mathfrak{A}}$ is not sufficient for $Z_{2}\left(\mathfrak{A}^{* *}\right) \hat{\mathfrak{A}} \subseteq \hat{\mathfrak{A}}$; iii) $Z_{1}\left(\mathfrak{A}^{* *}\right)=$ $Z_{2}\left(\mathfrak{A}^{* *}\right)=\hat{\mathfrak{A}}$ is not sufficient for $\mathfrak{A}$ to be weakly sequentially complete. These results answer three questions of Anthony To-Ming Lau and Ali Ülger.
\end{abstract}

Suppose that $\mathfrak{A}$ is a Banach algebra and let $\mathfrak{A}^{*}$ be the dual space of $\mathfrak{A}$. Then $\mathfrak{A}^{*}$ can be made into a Banach $\mathfrak{A}$-bimodule as follows: for $f \in \mathfrak{A}^{*}, a \in \mathfrak{A}, f a$ and af are defined by

$$
\begin{aligned}
& \langle f a, b\rangle=\langle f, a b\rangle, \\
& \langle a f, b\rangle=\langle f, b a\rangle \quad(b \in \mathfrak{A})
\end{aligned}
$$

where $\langle$,$\rangle is used for the dual pairing between elements of \mathfrak{A}^{*}$ and $\mathfrak{A}$.

Let $\mathfrak{A}^{*} \mathfrak{A}=\left\{f a: f \in \mathfrak{A}^{*}, a \in \mathfrak{A}\right\}$ and $\mathfrak{A A}^{*}=\left\{a f: a \in \mathfrak{A}, f \in \mathfrak{A}^{*}\right\}$. The dual space $\mathfrak{A}^{*}$ is said to factor on the left (resp. right) if $\mathfrak{A}^{*}=\mathfrak{A}^{*} \mathfrak{A}$ (resp. $\mathfrak{A}^{*}=$ $\left.\mathfrak{A A}^{*}\right)$. In a recent paper [5] Anthony To-Ming Lau and Ali Ülger have obtained various necessary and sufficient conditions for factoring of $\mathfrak{A}^{*}$. Here we answer three questions left open in [5].

The second dual space $\mathfrak{A}^{* *}$ of a Banach algebra $\mathfrak{A}$ admits two Banach algebra products known as first (left) and second (right) Arens products. Each of these products extends the product of $\mathfrak{A}$ as canonically embedded in $\mathfrak{A}^{* *}$ (see [1] or [3]). We briefly recall the definition of these products. For $m, n \in \mathfrak{A}^{* *}$, their first (left) Arens product indicated by $m \square n$ is given by

$$
\langle m \square n, f\rangle=\langle m, n f\rangle \quad\left(f \in \mathfrak{A}^{*}\right),
$$

where $n f \in \mathfrak{A}^{*}$ is defined by

$$
\langle n f, a\rangle=\langle n, f a\rangle \quad(a \in \mathfrak{A}),
$$

where $f a$ is as defined earlier.

Received by the editors December 5, 1996

1991 Mathematics Subject Classification. Primary 46H99.

Key words and phrases. Arens product, topological center, Banach module.

The first author was supported by NSERC grant OGP 003664 and the second author by NSERC grant A8069. 
The second Arens product of elements $m, n \in \mathfrak{A}^{* *}$ indicated by $m \diamond n$ is defined by

$$
\langle m \diamond n, f\rangle=\langle n, f m\rangle \quad\left(f \in \mathfrak{A}^{*}\right),
$$

where $f m$ is an element of $\mathfrak{A}^{*}$ defined by

$$
\langle f m, a\rangle=\langle m, a f\rangle \quad(a \in \mathfrak{A}) .
$$

Again we note that $a f$ is as defined earlier.

For each $n \in \mathfrak{A}^{* *}$, the mapping $m \mapsto m \square n$ (resp. $m \mapsto n \diamond m$ ) is weak*-weak* continuous. However for certain $n$, the mapping $m \mapsto n \square m$ (resp. $m \mapsto n \diamond m$ ) may fail to be weak* continuous. Due to this lack of symmetry the left (resp. right) topological center $Z_{1}\left(\mathfrak{A}^{* *}\right)$ (resp. $\left.Z_{2}\left(\mathfrak{A}^{* *}\right)\right)$ of $\mathfrak{A}^{* *}$ is defined by

$$
\begin{aligned}
& Z_{1}\left(\mathfrak{A}^{* *}\right)=\left\{m \in \mathfrak{A}^{* *}: n \mapsto m \square n \text { is weak }{ }^{*} \text {-weak }{ }^{*} \text { continuous }\right\}, \\
& Z_{2}\left(\mathfrak{A}^{* *}\right)=\left\{m \in \mathfrak{A}^{* *}: n \mapsto n \diamond m \text { is weak }{ }^{*} \text {-weak }{ }^{*} \text { continuous }\right\} .
\end{aligned}
$$

It follows easily from the definition of $Z_{1}\left(\mathfrak{A}^{* *}\right)$ (resp. $\left.Z_{2}\left(\mathfrak{A}^{* *}\right)\right)$ that $m \in Z_{1}\left(\mathfrak{A}^{* *}\right)$ (resp. $m \in Z_{2}\left(\mathfrak{A}^{* *}\right)$ ) if and only if $m \square n=m \diamond n$ (resp. $n \diamond m=n \square m$ ) for all $n \in \mathfrak{A}^{* *}$. In general $Z_{1}\left(\mathfrak{A}^{* *}\right)$ and $Z_{2}\left(\mathfrak{A}^{* *}\right)$ need not be equal (see [5], Example 2.5 and Remark 6.j, p.1211), but they both contain $\hat{\mathfrak{A}}$ ( $=$ the image of $\mathfrak{A}$ in $\mathfrak{A}^{* *}$ under the canonical mapping).

Anthony To-Ming Lau and Ali Ülger have shown in [5] that if $\mathfrak{A}^{*}$ factors on one side but not on the other, then $Z_{1}\left(\mathfrak{A}^{* *}\right) \neq Z_{2}\left(\mathfrak{A}^{* *}\right)$. In connection to this result they have asked:

Question. Suppose that $\mathfrak{A}$ has a bounded approximate identity. Does the equality $\mathfrak{A}^{*} \mathfrak{A}=\mathfrak{A A}^{*}$ imply that $Z_{1}\left(\mathfrak{A}^{* *}\right)=Z_{2}\left(\mathfrak{A}^{* *}\right)$ ?

We answer this question in the negative. In fact our counter example is a modification of one of the examples in [5].

For a Banach algebra $\mathfrak{A}$ we let $\mathfrak{A}^{\sharp}$ be the unitization of $\mathfrak{A}$. Thus $\mathfrak{A}^{\sharp}=\mathfrak{A} \oplus \mathbf{C}$, where $\mathbf{C}$ is the field of complex numbers. For $a, b \in \mathfrak{A}, \alpha, \beta \in \mathbf{C}$ we have $\|(a, \alpha)\|=$ $\|a\|+|\alpha|$ and $(a, \alpha)(b, \beta)=(a b+\alpha b+\beta a, \alpha \beta)$.

1. Lemma. For any Banach algebra $\mathfrak{A}, Z_{1}\left(\left(\mathfrak{A}^{\sharp}\right)^{* *}\right)=Z_{1}\left(\mathfrak{A}^{* *}\right) \oplus \mathbf{C}$ and a similar result holds for the right topological centers.

Proof. First we note that the two Banach algebras $\left(\mathfrak{A}^{\sharp}\right)^{* *}$ and $\left(\mathfrak{A}^{* *}\right)^{\sharp}$ are isomorphic, when they both have the first Arens product. Then the rest is straightforward from the definition of topological centers.

The following answers question (6d) in [5].

2. Theorem. There exists a Banach algebra $\mathfrak{A}$ possessing a bounded approximate identity and satisfying $\mathfrak{A}^{*} \mathfrak{A}=\mathfrak{A A}^{*}=\mathfrak{A}^{*}$, but $Z_{1}\left(\mathfrak{A}^{* *}\right) \neq Z_{2}\left(\mathfrak{A}^{* *}\right)$.

Proof. It is known that there exists a Banach algebra $\mathfrak{B}$ having a bounded approximate identity for which $Z_{1}\left(\mathfrak{B}^{* *}\right) \neq Z_{2}\left(\mathfrak{B}^{* *}\right)$ (see [5]). Then for $\mathfrak{A}=\mathfrak{B}^{\sharp}$, by Lemma 1 we have $Z_{1}\left(\mathfrak{A}^{* *}\right) \neq Z_{2}\left(\mathfrak{A}^{* *}\right)$. However, $\mathfrak{A}^{*} \mathfrak{A}=\mathfrak{A}^{*}=\mathfrak{A}^{*}$, since $\mathfrak{A}$ is unital.

For the origin of the following question see [5, question 6e].

Question. Does the inclusion $\hat{\mathfrak{A}} Z_{1}\left(\mathfrak{A}^{* *}\right) \subseteq \hat{\mathfrak{A}}$ imply $Z_{2}\left(\mathfrak{A}^{* *}\right) \hat{\mathfrak{A}} \subseteq \hat{\mathfrak{A}}$ ? 
To answer this question, we first recall that if $\mathfrak{A}$ is a Banach algebra then $\mathfrak{A}^{o p}$ is the algebra obtained by reversing the order of multiplication in $\mathfrak{A}$; i.e., $\mathfrak{A}^{o p}$ has the product $\circ$ given by $a \circ b=b a$, for every $a, b \in \mathfrak{A}$.

3. Theorem. There exists a Banach algebra $\mathfrak{A}$ with a bounded approximate identity for which $\hat{\mathfrak{A}} Z_{1}\left(\mathfrak{A}^{* *}\right) \subset \hat{\mathfrak{A}}$, but $Z_{2}\left(\mathfrak{A}^{* *}\right) \hat{\mathfrak{A}}$ is not contained in $\hat{\mathfrak{A}}$.

Proof. There exists a Banach algebra $\mathfrak{B}$, with a bounded approximate identity, such that $Z_{2}\left(\mathfrak{B}^{* *}\right)=\hat{\mathfrak{B}}$ but $Z_{1}\left(\mathfrak{B}^{* *}\right)$ is larger than $\hat{\mathfrak{B}}$ (see [5]). It is well known that switching to the opposite multiplication in a Banach algebra results in interchanging the first and the second Arens products in its second dual space. From this and by Lemma 1 , for $\mathfrak{A}=\left(\mathfrak{B}^{o p}\right)^{\sharp}$ we have

$$
Z_{1}\left(\mathfrak{A}^{* *}\right)=\hat{\mathfrak{B}^{o p}} \oplus \mathbf{C}=\hat{\mathfrak{A}}
$$

and

$$
Z_{2}\left(\mathfrak{A}^{* *}\right)=Z_{1}\left(\mathfrak{B}^{* *}\right) \oplus \mathbf{C}
$$

Hence

$$
\hat{\mathfrak{A}} Z_{1}\left(\mathfrak{A}^{* *}\right)=\left(\hat{\mathfrak{B}^{o p}} \oplus \mathbf{C}\right)\left(\hat{\mathfrak{B}^{o p}} \oplus \mathbf{C}\right)=\hat{\mathfrak{B}^{o p}} \oplus \mathbf{C}=\hat{\mathfrak{A}}
$$

and

$$
Z_{2}\left(\mathfrak{A}^{* *}\right) \hat{\mathfrak{A}}=\left(Z_{1}\left(\mathfrak{B}^{* *}\right) \oplus \mathbf{C}\right)\left(\hat{\mathfrak{B}^{o p}} \oplus \mathbf{C}\right)=Z_{1}\left(\mathfrak{B}^{* *}\right) \oplus \mathbf{C} .
$$

But $Z_{1}\left(\mathfrak{B}^{* *}\right) \oplus \mathbf{C}$ is larger than $\hat{\mathfrak{A}}$, since $Z_{1}\left(\mathfrak{B}^{* *}\right)$ is larger than $\hat{\mathfrak{B}}$.

In [5], Lau and Ülger ask (question $6 \mathrm{j})$ : if $Z_{1}\left(\mathfrak{A}^{* *}\right)=Z_{2}\left(\mathfrak{A}^{* *}\right)=\hat{\mathfrak{A}}$, must $\mathfrak{A}$ be weakly sequentially complete?

We give an example of a Banach algebra $\mathfrak{A}$ with identity, and such that $Z_{1}\left(\mathfrak{A}^{* *}\right)=$ $Z_{2}\left(\mathfrak{A}^{* *}\right)=\hat{\mathfrak{A}}$, but with $\mathfrak{A}$ not weakly sequentially complete. First we describe a general construction; this construction is known and has been used by workers in the area of Automatic Continuity (see [2], p. 647).

Let $A$ be a Banach algebra, and let $M$ be a Banach $\mathfrak{A}$-bimodule. Then $\mathfrak{A}=M \oplus$ $A$, with norm $\|(m, a)\|=\|m\|+\|a\|$, and product $(m, a)(n, b)=(m b+a n, a b)$ is a Banach algebra. If $A$ has a bounded approximate identity $\left(e_{j}\right)$ and if $M$ factors $(M=M A=A M)$, then $\left(0, e_{j}\right)$ is a bounded approximate identity for $\mathfrak{A}$. In particular, if $A$ has an identity $e$, and $M$ is unital, then $(0, e)$ is an identity for $\mathfrak{A}$. Note that $\mathfrak{A}^{*}=M^{*} \times A^{*}$ (with norm $\|(s, f)\|=\max \{\|s\|,\|f\|\}$ ), and that $\mathfrak{A}^{* *}=M^{* *} \oplus A^{* *}$. It is routine to check that $\mathfrak{A}$ is weakly sequentially complete if and only if each of $A$ and $M$ is weakly sequentially complete. Also, it can be checked that the first Arens product on $\mathfrak{A}^{* *}$ satisfies

(1) $(\mu, \alpha) \square(\nu, \beta)=(\mu \square \beta+\alpha \square \nu, \alpha \square \beta)$, for $\mu, \nu \in M^{* *}$ and $\alpha, \beta \in A^{* *}$.

Here, in all cases, $\square$ has been used to denote that extension of a bilinear operation to second duals, which is weak*-weak* continuous in the left-hand argument; for instance, $(\mu, \beta) \mapsto \mu \square \beta$ denotes the extension to $M^{* *} \times A^{* *}$ of the right module action $(m, b) \mapsto m b$ of $A$ on $M$ which is weak*-weak* continuous in $\mu$.

Now we identify $Z_{1}\left(\mathfrak{A}^{* *}\right)$. Since a net $\left(\nu_{j}, \beta_{j}\right)$ in $\mathfrak{A}^{* *}$ converges weak ${ }^{*}$ to $(\nu, \beta)$ if and only if $\nu_{j} \rightarrow \nu$ weak ${ }^{*}$ in $M^{* *}$ and $\beta_{j} \rightarrow \beta$ weak* in $A^{* *}$, it is straightforward to check that $(\mu, \alpha) \in Z_{1}\left(\mathfrak{A}^{* *}\right)$ if and only if

(2) $\alpha \in Z_{1}\left(A^{* *}\right)$;

(3) $\nu \mapsto \alpha \square \nu$ is weak*-weak* continuous on $M^{* *}$; and

(4) $\beta \mapsto \mu \square \beta$ is weak ${ }^{*}$-weak ${ }^{*}$ continuous $A^{* *} \rightarrow M^{* *}$. 
Now suppose $Z_{1}\left(A^{* *}\right)=\hat{A}$. Then (2) implies $\alpha \in \hat{A}$, and it is then straightforward to verify that (3) holds. So in this case, $(\mu, \alpha) \in Z_{1}\left(\mathfrak{A}^{* *}\right)$ if and only if $\alpha \in \hat{A}$ and (4) holds.

Finally, we consider the particular case $M=A^{*}$. Recall that we are assuming $A$ has a bounded approximate identity. To ensure that $\mathfrak{A}^{* *}$ has a bounded approximate identity, we suppose that $A^{*}$ factors, in the sense of [5]. Note that the case that $A$ has an identity is included.

Now we have $M^{*}=A^{* *}$ and $M^{* *}=A^{* * *}$, and the meanings of the various bilinear operations have to be carefully distinguished; however, it can be shown that, for $\mu \in M^{* *}, \beta \in A^{* *}$, and $\alpha \in M^{*}=A^{* *}$,

$$
\langle\mu \square \beta, \alpha\rangle=\langle\mu, \beta \square \alpha\rangle ;
$$

that is, the bilinear map $(\mu, \beta) \mapsto \mu \square \beta$ on $M^{* *} \times A^{* *}$ to $M^{* *}$ is the right dual module action of $A^{* *}$ on $A^{* * *}=M^{* *}$ corresponding to the first Arens product on $A^{* *}$. Suppose (4) holds, and take $\alpha=E$, a right identity for the first Arens product in $A^{* *}=M^{*}$. If $\beta_{j} \rightarrow \beta$ weak* in $A^{* *}$, then by (4),

$$
\left\langle\mu, \beta_{j}\right\rangle=\left\langle\mu, \beta_{j} \square E\right\rangle=\left\langle\mu \square \beta_{j}, E\right\rangle \rightarrow\langle\mu \square \beta, E\rangle=\langle\mu, \beta\rangle .
$$

That is, (4) implies that $\mu$ is a weak*- continuous linear functional on $A^{* *}$, so that $\mu \in\left(A^{*}\right)^{r}=\hat{M}$. The converse is easy, so we have proved the conclusion $Z_{1}\left(\mathfrak{A}^{* *}\right)=\hat{M} \oplus \hat{A}=\hat{\mathfrak{A}}$ in the following proposition; the arguments for $Z_{2}\left(\mathfrak{A}^{* *}\right)$ are quite similar.

6. Proposition. Let $A$ be a Banach algebra with a bounded approximate identity.

(1) Assume $A^{*}$ factors (in the sense of [5]). Then $\mathfrak{A}=A^{*} \oplus A$ has a bounded approximate identity.

(2) Assume $Z_{1}\left(A^{* *}\right)=Z_{2}\left(A^{* *}\right)=\hat{A}$. Then $Z_{1}\left(\mathfrak{A}^{* *}\right)=Z_{2}\left(\mathfrak{A}^{* *}\right)=\hat{\mathfrak{A}}$.

(3) $\mathfrak{A}$ is weakly sequentially complete if and only if each of $A$ and $A^{*}$ is weakly sequentially complete.

If we take $A=l^{1}(\mathbb{Z})$ (the group algebra of the discrete group of integers), then $Z_{1}\left(A^{* *}\right)=Z_{2}\left(A^{* *}\right)=\hat{A}$ (see [4]), and all the assumptions above are satisfied; in particular, $A$ has an identity. Also, $A^{*}=l^{\infty}(\mathbb{Z})$ is not weakly sequentially complete. So $\mathfrak{A}=A^{*} \oplus A$ has an identity, satisfies $Z_{1}\left(\mathfrak{A}^{* *}\right)=Z_{2}\left(\mathfrak{A}^{* *}\right)=\hat{\mathfrak{A}}$, and $\mathfrak{A}$ is not weakly sequentially complete. That answers (negatively) question $6 \mathrm{j}$ of [5].

\section{REFERENCES}

[1] R. Arens, 'The adjoint of a bilinear operation', Proc. Amer. Math. Soc. 2 (1951), 839 - 848. MR 13:659f

[2] H.G. Dales, 'The uniqueness of the functional calculus', Proc. London Math. Soc. (3) 27 (1973), 638-648. MR 48:12062

[3] J. Duncan and S.A.R. Hosseiniun, 'The second dual of a Banach algebra', Proc. Roy. Soc. Edinburgh Sect. A84 (1979), 309 - 325. MR 81f:46057

[4] A.T. Lau and V. Losert, On the second conjugate algebra of $L^{1}(G)$ of a locally compact group, J. London Math Soc. 37 (1988), 464-470. MR 89e:43007

[5] A.T. Lau and A. Ülger, 'Topological centers of certain dual algebras', Trans. Amer. Math. Soc. vol. 348, no. 3 (1996), 1191 - 1212. MR 96h:43003

Department of Mathematics and Astronomy, University of Manitoba, Winnipeg R3T 2N2, CANADA 